\title{
The Midcourse Space Experiment (MSX)
}

\author{
Bruce D. Guilmain \\ Ballistic Missile Defense Organization \\ Pentagon (rm 1E168), Washington D.C. 20301-7100 \\ (703)697-4025, Guilmain@tecnetl.jcte.jcs.mil
}

Abstract--The Midcourse Space Experiment (MSX) is designed and built around an infrastructure of eight scientific teams; it will be the first and only extended duration, multiwavelength $(0.1$ to $28 \mu \mathrm{m})$ phenomenology measurement program funded and managed by Ballistic Missile Defense Organization (BMDO). During its 16 month cryogen lifetime and five year satellite lifetime, MSX will provide high quality target, earth, earthlimb, and celestial multi-wavelength phenomenology data. This data is essential to fill critical gaps in phenomenology and discrimination data bases, furthering development of robust models of representative scenes, and assessing optical discrimination algorithms. The MSX organization is comprised of self-directed work teams in six functional areas. These teams provide a unique blend of scientists and engineers from academia and industry. Experiments formulated by each of the eight scientific teams will be executed on the satellite in a $903 \mathrm{~km}$ near polar orbit $\left(99.23^{\circ}\right.$ inclination), with an eccentricity of 0.001 , argument of perigee of 0 , and the right ascension of the ascending node is 250.0025 . Two dedicated target missions are planned consisting of one Strategic Target System launch out of Barking Sands, Hawaii, and two Low Cost Launch Vehicles launches out of Wallops Island, VA. These target missions will deploy various targets, enabling the MSX principal investigator teams to study key issues such as metric discrimination, deployment phase tracking, cluster tracking, fragment bulk filtering, tumbling re-entry vehicle signatures, etc. A data management infrastructure to ensure that the data is processed, analyzed, and archived will be available at launch time. The raw data and its associated calibration files and software will be archived, providing the customer with a cataloged database containing verified, validated, and carefully calibrated data. This paper describes the MSX program objectives, target missions, data management architecture, and organization.

\section{TABLE OF CONTENTS}

1. INTRODUCTION

2. PROGRAM OVERVIEW

3. SATELLITE

4. SCIENTIFIC TEAMS

5. TARGETS

6. OPERATIONS

7. DATA MANAGEMENT

8. ORGANIZATION

9. SUMMARY 


\section{INTRODUCTION}

There are generic issues for any passive sensor that is required to track and discriminate dim targets against cluttered backgrounds. In the early days of the Strategic Defense Initiative data requirements were identified to address these issues and to fill critical gaps in phenomenology and discrimination databases, validate and enhance models, and improve algorithms.

Based on these requirements a data gathering and measurements system, MSX, was designed, built, and is currently being integrated and tested. The Midcourse Space Experiment is a multi-year space demonstration and data collection experiment addressing three main objectives for the Ballistic Missile Defense Organization:

- Functionally demonstrate the capability of midwave infrared, longwave infrared, ultraviolet, and visible sensors to acquire, track, and discriminate objects associated with the midcourse (after booster burnout and before reentry) phase of a ballistic missile flight, and of resident space objects.

- Collect a statistically significant natural phenomenology and target signature database to improve and validate models and serve as a system design database.

- Validate key sensor technologies in operational environments over extended periods to support technology transfer. Evaluate extended on-orbit performance data on focal planes, optics, and processors.

The MSX program has assembled a technical group containing about 100 leading DOD community experts. These people have been participating in the design and development of MSX from the inception of the program. The group, representing about 30 industry and government organizations has the broad background and depth of experience required to assure that the MSX program meets evolving national needs.

In the process of meeting the BMDO objectives MSX will also contribute significantly to the understanding of scientific issues of national interest such as global change, remote sensing, astronomy, astrophysics, and orbital debris.

The MSX "observatory" style satellite is scheduled to begin its five year mission in early 1996 with a launch on a Delta II booster from Vandenberg Air Force Base, CA.

\section{PROGRAM OVERVIEW}

The Midcourse Space Experiment will be the first extended duration, multi-wavelength phenomenology measurements program sponsored by BMDO. The MSX program will accomplish its mission by conducting a series of experiments over a period of five years. This five year period is divided into two phases; a cryogen phase and a post cryogen phase. The cryogen phase, the period in which there is cryogen required by the infrared sensor, is expected to last 16 months.

As experiments are executed on the satellite in its orbit data will be stored on two 54 Gbit recorders. Data will be downloaded via a 25 Mbit per second link to Johns Hopkins University's Applied Physics Laboratory. The Mission Operations Center located at JHU/APL contains the primary Mission Control Center, the MSX Tracking Station, the Mission Processing Center the Attitude Processing Center, and the Operations Planning Center. The Mission Control Center commands, controls, and monitors the satellite's state of 
health. The Mission Processing Center receives the raw science data and distributes the data to the sensor vendors, the Principal Investigators (PIs), and BMDO's Background Data Center at the Naval Research Laboratory. The vendors will verify the data by ensuring that their instruments are operating correctly. The PIs will validate the data by analyzing the results of their experiments. A Data Certifying and Technology Transfer Team certifies the data by providing an "independent" oversight of the entire process from sensor fabrication, calibration (on the ground and on orbit) through software processing of the science data. All MSX data (science data, calibration records, certified software and final calibration factors) will be archived at the Background Data Center. The Background Data Center maintains an on-line catalog to aid in data selection.

\section{SATELLITE}

The MSX satellite (Figure 1) structure consists of three main sections: the instrument section, the truss structure, and the electronics section. The instrument section houses 11 optical sensors, which are precisely aligned so that target activity can be viewed simultaneously by multiple sensors. MSX is capable of observations at a wide range of infrared, visible, and ultraviolet wavelengths. To maintain coalignment of the sensors, heat pipes are embedded in the aluminum honeycomb panels to keep the temperature even throughout the entire instrument section. A midsection graphite epoxy truss supports the large cryogenic dewar, which contains frozen hydrogen at approximately $9^{\circ}$ $\mathrm{K}$. The $200 \mathrm{~cm}$ long truss thermally isolates the heat-sensitive instrument section from the

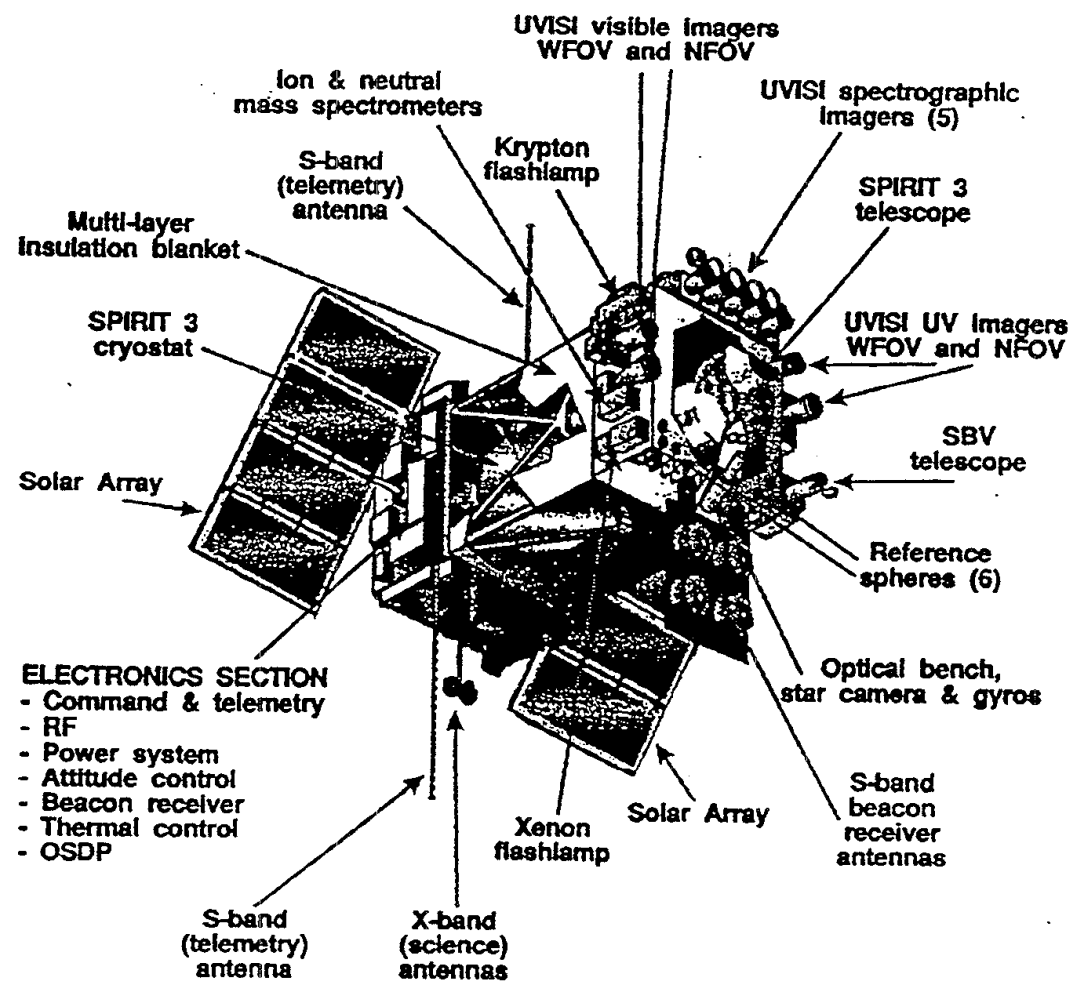

Figure 1. MSX Satellite 
electronic section. Three sides of the truss are covered with multilayer insulation to shield the dewar from the sun and Earth. The attitude control hardware consists of four reaction wheels and three magnetic torque rods. Any three of the four wheels can provide 3-axis control of the spacecraft. Attitude sensors include two 3-axis ring laser gyro systems, a star camera, two horizon sensors, five digital sun angle detectors, and a 3-axis magnetometer. The electronic section carries the warm electronics that are a part of all the instruments. Placing the warm electronics in this section minimizes thermal dissipation in the instrument section allowing the cryogenically cooled instruments to operate as cold as possible. The spacecraft weighs $2800 \mathrm{~kg}$ and excluding the two solar arrays measures $510 \mathrm{~cm}$ in length with a $150 \mathrm{~cm}$ by $150 \mathrm{~cm}$ cross section. Raw sensor data is recorded onboard the spacecraft with full fidelity, and later transmitted to the primary ground site over an X-band 25Mbps downlink. Commands are uplinked and satellite housekeeping data downlinked in real time over an S-band link, to either the primary ground site or the Air Force Satellite Control Network. Commands may be executed in near-real time, but are normally stored for execution at a later time. The precise pointing knowledge required by the mission has lead to stringent requirements on the spacecraft for attitude determination, control and stability. The system achieves real-time pointing accuracy of better than $0.1^{\circ}$ and post-processing knowledge to 9 microradians. Line-of-sight jitter is held to 9 microradians over instrument integration durations of approximately 1 second. The spacecraft is three-axis stabilized with reaction wheels to provide high precision pointing and maximize sensor performance by not introducing contaminates into the sensor environment. Because there are no expendables on the spacecraft bus (such as propellants), lifetime is limited only by the reliability of the individual subsystems. The spacecraft is powered by a combination of solar panels and a high-capacity, $\mathrm{NiH} 2$ battery. This system is designed to deliver $2.5 \mathrm{kw}$. During data collection events approximately $50 \%$ of the available power is used by the instruments. The primary instrument systems include the SPace InfRared Imaging Telescope (SPIRIT III), Ultraviolet and Visible Imagers and Spectrographic Imagers (UVISI), Space-Based Visible (SBV) instrument, an Onboard Signal and Data Processor, and contamination sensors.

SPIRIT III a cryogenically cooled infrared sensor is the most advanced infrared instrument yet launched into space. In th elong wavelength SPIRIT III has approximately the same sensitivity but 30 times better spatial resolution than the Infrared Astronomy Satellite. ${ }^{1}$ Developed by the Space Dynamics Laboratory of Utah State University, SPIRIT III includes a five-color, high-spatial-resolution scanning radiometer and a six-channel, high-spectralresolution, fourier-transform spectrometer. SPIRIT III is the primary sensor for target and background data collection. Its key features include rejection of light from sources outside the field of view, and high spatial and spectral resolution. The infrared radiometer and interferometer detector bandwidths were chosen to address particular data collection needs, including: thermal discrimination, cold target detection, earthlimb clutter measurement, and atmospheric composition measurement.

The Ultraviolet and Visible Imagers and Spectrographic Imagers, is a Johns Hopkins University/Applied Physics Laboratory built instrument with five spectrographic imagers, and four ultraviolet and visible imagers. UVISI provides complete spectral and imaging capabilities from the far ultraviolet through 
visible wavelengths.

The Space-Based Visible instrument, equipped with a charged-coupled device, is a visible band telescope with a six-inch aperture and image processing electronics. Built by MIT/Lincoln Laboratory, the space-based visible sensor will demonstrate an above-the-horizon surveillance capability in visible wavelengths from a space platform. The SBV sensor will also supplement the target and background phenomenology data collected by SPIRIT III and UVISI. The spectral coverage of SPIRIT III, UVISI, and SBV is illustrated in Figure 2. view. These sensors were chosen to measure specific contaminants (such as water vapor) in the spacecraft environment. These measurements will validate the strict contamination control plan followed throughout the development of the satellite, enhance satellite contamination models, and measure contaminants in situ.

\section{SCIENTIFIC TEAMS}

The Chief Scientist leads the experiment planning function. He interprets the MSX

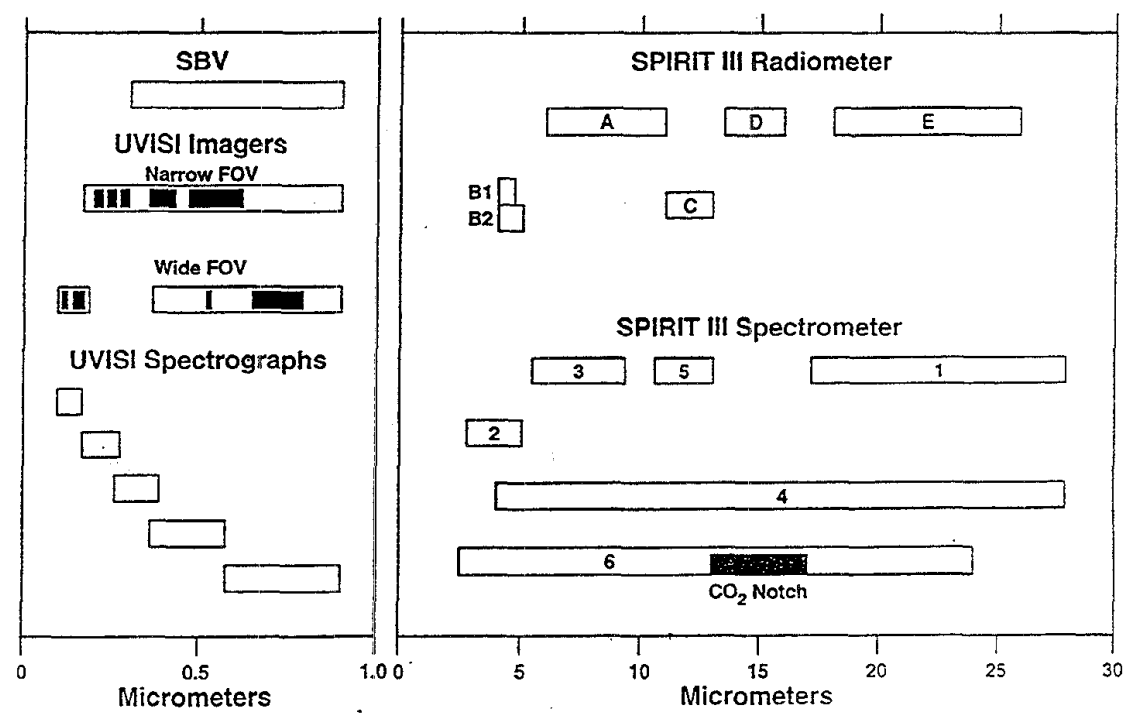

Figure 2. MSX Spectral Coverage

The On-board Signal and Data Processor was built by Hughes Aircraft $\mathrm{Co}$. and uses data from SPIRIT III in real-time signal processing for target detection and tracking. It also will provide information about radiation effects on state-of-the-art semiconductor devices.

The contamination sensors include five quartz crystal microbalances, a pressure sensor, neutral and ion mass spectrometers, and flash lamps to illuminate contaminants in the sensor fields-of- science objectives in light of evolving BMDO and science community requirements. The Chief Scientist also chairs the PI executive committee. This group reviews experiment plans, coordinates with the Mission Planning Team and supporting organizations to execute the experiments, and certifies the analyzed results of the experiments. Each PI and his team of experts from various organizations are responsible for defining the science and modeling requirements in their category, 
designing the experiments, and analyzing the resulting data to satisfy MSX mission objectives and requirements. A brief "mission" objective for each of the eight PI teams follows.

The Early Midcourse Target team concentrates on the phenomenology associated with the boost through deployment phase of ballistic missiles. This team will address target acquisition, deployment, and tracking against cluttered backgrounds, as well as metric discrimination. This PI will use the dedicated target missions as the primary source of data. Targets of Opportunity will be used to supplement this data.

The Theater and Midcourse Cooperative Target team will concentrate on the latter phase of a missile's exoatmospheric trajectory and will demonstrate tracking and handover functions on credible targets. This PI is concerned with payload evolution from post-deployment through reentry. This team will focus on thermal and dynamic discrimination, and target signature collection.

Terrestrial, earthlimb, and celestial backgrounds influence target measurements taken against a cluttered environment. PI teams will make dedicated background measurements over a variety of conditions to provide real data to evaluate their impact on sensors and overall system performance. Atmospheric background measurements will be collected as a function of tangent altitude, latitude, season, and atmospheric conditions. The goal is to establish the characteristics of small scale spatial irregularities of earth and earthlimb backgrounds and to determine their global distribution, associations with certain phenomena, and frequencies of occurrence. Experiments will measure and characterize the effect of celestial structures and moving backgrounds, earth radiance, and atmospheric signal attenuation existing at low elevation angles, and also measure photon noise generated by zodiacal background.

The Earthlimb/Aurora team will obtain ultraviolet through very longwave infrared data which is required for evaluation of missile defense system performance. Earthlimb experiments will focus primarily on measuring all natural disturbances from pole to pole over all local times and seasons. Specific emphasis will be placed on measuring earthlimb radiance, auroral emissions intensities, radiance, and structure, and on the spatial distribution and IR radiance of mesospheric clouds and terrestrial clutter.

The Celestial Backgrounds team will characterize representative and stressing celestial backgrounds. The results of the celestial background experiments will upgrade the brightness/resolution databases to satisfy strategic defense system requirements. Celestial background measurements will be providing a comprehensive survey of the stellar sky with emphasis on the solar system structure, detail, and point sources.

The Shortwavelength Terrestrial Backgrounds team has a primary science mission to characterize the terrestrial auroral and airglow limb and below-the-horizon spectral databases in the 110 to $900 \mathrm{~nm}$ wavelength range using the UVISI instrument. The goal is to acquire a representative database on global, seasonal, diurnal, and temporal variations. The Terrestrial backgrounds experiments obtain shortwave data which supports strategic defense system functions performed against stressing terrestrial backgrounds.

Space Surveillance team's experiments will 
provide a functional demonstration of the spacebased surveillance capability and address the detection of space targets against stressing backgrounds. This analysis will focus on surveillance sensor performance, network integration, critical issues for space surveillance, and applications to future systems. MSX will be the first space-based platform to perform space surveillance demonstrations.

The Contamination team has overview of contamination control and monitoring through the life of the program. They oversee material choice and handling during hardware development and integration, through the contamination control plan. This plan will be validated by on-orbit data and will be a legacy to future satellite programs. The contamination team is responsible for monitoring, modeling and documenting the effects of contamination (from the spacecraft and from the ambient environment) on optical sensors. The contamination experiments quantify contamination effects on optical sensor performance. The contamination team will update pre-launch models developed for use in predicting obscurants, measure in-flight contaminates, and characterize particulate and molecular contamination in the space environment which impair the functioning of space-based sensors and limit their effective lifetime.

The Data Certification and Technology Transfer (DCATT) team oversees the calibration of the sensors, certifies the quality of the data, and transfers the results of the technology demonstrations and lessons learned. The DCATT team represents a unique approach to sensor characterization. Pre-launch, the DCATT team works with each sensor vendor to develop, implement, and document a sensor calibration plan. They also work together to develop a set of algorithms to calibrate the raw sensor data, and develop an automated process for verifying the quality of the data. This allows the production of high quality calibrated data in an automated, repeatable fashion.

The certification technique used is similar to a method of process certification used in manufacturing. The DCATT team will review the calibration and performance of the sensors on the ground by bounding the expected onorbit operating conditions within an envelope. Within these bounds, the process by which the raw data is converted to engineering units will be certified by the DCATT team. MSX is a scientific data gathering and measurements program and great care has been taken to understand each instrument and its calibration requirements. Calibration on the ground and onorbit with reference spheres are all traceable to National Institute of Standards and Technology. The DCATT team oversees the implementation of a comprehensive instrument calibration plan, guidance of the data certification process, and distribution of technological and programmatic information once it has been certified. The DCATT team will determine the metric and photometric accuracy and precision of the sensors, biases in the metric data and absolute photometric calibration. The DCATT team will also characterize the metric error and the photometric precision, provide geometric and photometric calibrations and additionally provide traceability to sensor measurements- a means to investigate problems with on-orbit sensor performance. DCATT activities will enable MSX to define actual sensor performance capabilities. The DCATT team provides a key interface between the individual PI tearns and the sensor vendors.

\section{TARGETS}


The Targets Teams develops and procures target objects, instrumentation and launch vehicles for the MSX dedicated target experiments. They test and characterize the target objects. They are also responsible for ensuring the targets are ready for launch when required, and conducting launch operations. The U.S. Army Strategic Defense Command executes the Strategic Target System (STARS) mission and directs target development at Sandia National Laboratories for the STARS and Low Cost Launch Vehicle missions. The Air Force's National Aerospace Intelligence Center executes the Low cost Launch Vehicle program.

The dedicated targets include a number of test objects deployed from the Operational Deployment Experiment Simulator, launched on the Strategic Target System booster. One dedicated target will be launched from the Kauai Test Facility in Kauai, Hawaii, and impact in the broad ocean area north of the US Army Kwajalein Atoll. The Post Boost Vehicle launched on the STARS II booster will deploy twenty-six midcourse objects. These 26 objects represent a number of different target types and deployment techniques. All the target objects will be deployed in sunlight and will subsequently cross the Earth's shadow into darkness. Two Low Cost Launch Vehicles will be launched out of Wallops Island, VA. Each of these sounding rockets will carry at least five highly characterized targets.

These target missions will lead to the development of an extensive database of midcourse target signature phenomenology observed from a space-based sensor.

\section{OPERATIONS}

MSX satellite operations are carried out by cooperating teams from several organizations. These teams are responsible for scheduling and executing spacecraft activities, collecting the data, and maintaining the health of the spacecraft.

The Mission Planning Team validates the mission requirements, determines how each experiment fits in the mission priorities, and develops a master plan for performing experiments to satisfy the mission requirements. The Mission Planning Team provides the Operations Planning Team with monthly objectives based on the master plan, the OPT analyzes each experiment to ensure it can be accomplished without violating spacecraft constraints, and then schedules the experiments to meet the monthly objectives. The SBV Payload Operations and Control Center participates in this process for surveillance experiments. The Operations Planning Team then produces the set of commands needed to run all scheduled spacecraft activities, and hands them off to the control team. The Mission Operations Center is the primary ground site for MSX. The Operations Control Team is responsible for uplinking the commands to the spacecraft, downlinking the recorded data and real time data, and monitoring the spacecraft health and status. Uplinks and downlinks may be directly between the Mission Operations Center and the spacecraft, or they may go through the Air Force Satellite Control Network (recorded data can only be received at the Mission Operations Center, because of the high data rate). The Test Support Center is the secondary ground site, and has the capability of commanding the spacecraft if necessary. The On-orbit Spacecraft Performance Assessment Team and the sensor performance assessment teams monitor spacecraft and sensor performance, and identify, diagnose, and resolve problems with the spacecraft and sensors. The 
Mission Planning Team and Operations Planning Teams work together to schedule experiments in a way to optimize satisfaction of mission requirements. The Operations Control Team maintains the health of the satellite, executes the experiments, and collects the data.
The MSX Data Management system has been designed to swiftly flow the data to the PI teams. Data processing tasks are distributed so that experts may do their part at their home institutions. Data Management responsibilities extend from telemetry processing through data

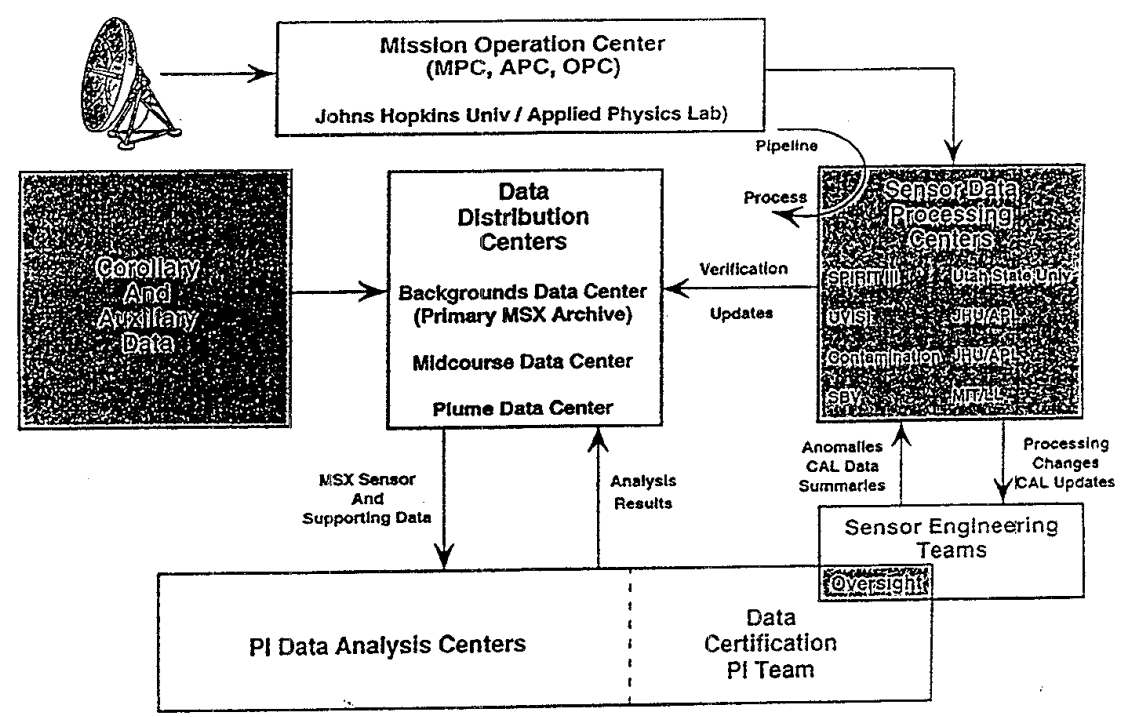

Figure 3. MSX Sensor Data flow

The Mission Planning Team consists of the Program Manager, Chief Scientist, Technical Advisor, System Engineer, and representatives from user organizations. The MPT provides top-level mission planning, establishes experiment priorities, approves and maintains configuration control of experiment plans, and approves monthly operations planning. Satellite resources required to execute each experiment are estimated by the operational planning team in close coordination with the PIs and the sensor vendors. This enables the MPT to assess the "cost" versus the benefit of each experiment, and helps ensure the most efficient utilization of the satellite's resources. This planning is vital during the cryogen phase of the mission.

\section{DATA MANAGEMENT}

reduction, and to distributing the data products to the scientist and other users. Initial processing is done at the APL Mission Processing Center. Tapes are sent simultaneously to the Data Processing Centers (one for each sensor) and the data archive and distribution centers. The Data Processing Centers monitor the quality of the data and maintain the calibration software and associated calibration products. Calibration and verification of data is done by the sensor vendors at the Data Processing Centers. This data verification is done using an automated "pipeline" process which is certified by the DCATT PI team. Data analysis and validation are done by the Principal Investigator teams. Data certification is done by the DCATT PI Team with participation of the sensor vendors and PIs. The primary archive and distribution center is the Backgrounds Data 
Center, at the Naval Research Laboratory. The data center is also responsible for acquiring supporting data, collecting pre-launch testing and calibration data, and archiving all PI analysis products. Due to the volume of data anticipated from this program a "pipeline" data management architecture has been implemented in which automated tools are used whenever possible to minimize any processing delays. The Data Center archive all mission data and analysis products, and automatically distribute data to the Data Analysis Centers, including science and housekeeping data from the Mission Operations Center, and data quality indicators from the Data Processing Centers. The archive data are available upon request to the broader DOD and science community, with proper clearance and approval from the MSX Program Manager. A top level MSX sensor data flow diagram is presented in Figure 3.

\section{ORGANIZATTION}

The MSX program is comprised of self-directed work teams in six broad functional areas:
Principal Investigators, spacecraft and ground operations, sensors, targets, and target operations.

The MSX program has over 300 individuals from more than 30 organizations. Actual implementation of technical direction was left to the self directed work team with assistance and lessons learned passed on from other teams in the MSX organization. A collective decision making philosophy is exercised in which individuals are involved in decisions which could affect their area of responsibility. Program management has focused on the financial, technical, and schedule constraints bounding each team's environment, and has ensured that all interfaces between organizations are met and controlled.

Managed by the Ballistic Missile Defense Organization and executed by universities, the MSX program has no prime contractor. The principal organizations involved in key program elements are listed below and illustrated in Figure 4.

The spacecraft bus, spacecraft integration,

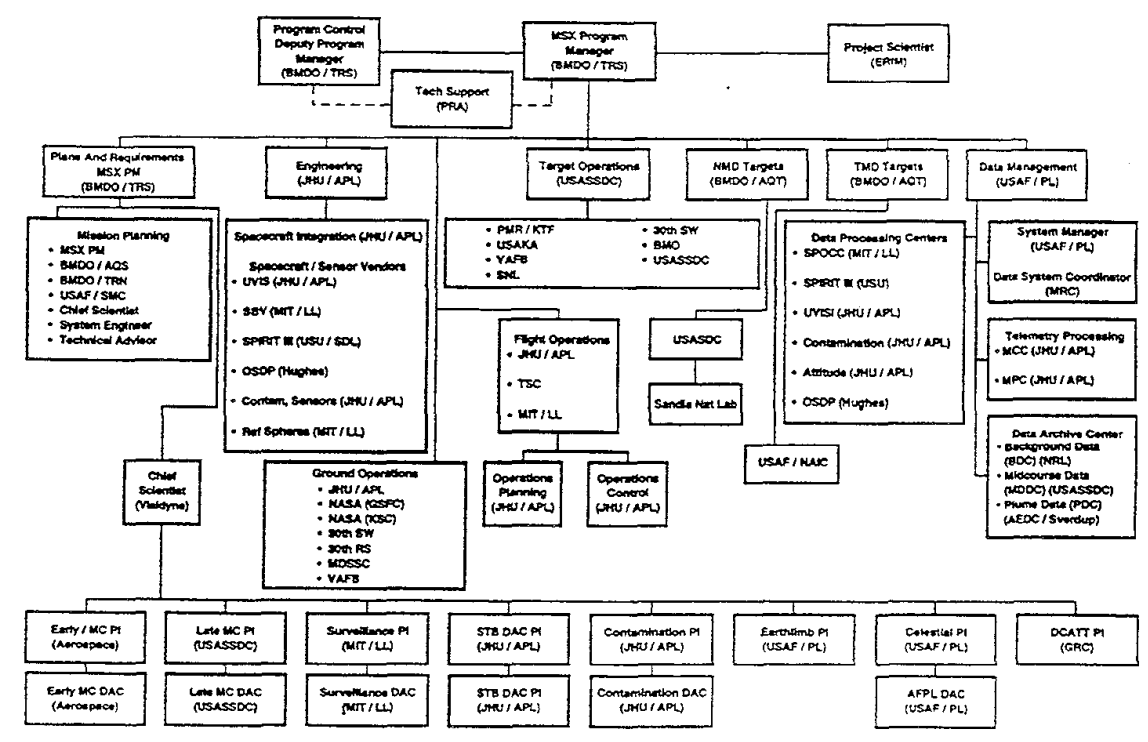

Figure 4. MSX Organization 
ultraviolet and visible imagers and spectrographic imager instruments, contamination instruments, and operations support are provided by the Johns Hopkins University/Applied Physics Laboratory .

Utah State University provides the Spatial Infrared Imaging Telescope. Hughes Corporation is providing the Onboard Signal Data Processor.

The Space-based visible instrument and the corresponding payload operations Center are provided by MIT/Lincoln Laboratory

McDonnell Douglas Aerospace Company will be providing the Delta II booster.

\section{SUMMARY}

The MSX satellite has a suite of state-of-the-art sensors covering the spectrum from the far ultraviolet to the very longwave infrared. It will provide data to answer fundamental questions about the performance of BMDO surveillance systems, and provide environmental data of global interest.

MSX will minimize risk in the development of critical National Missile Defense and long-range theater missile defense systems by collecting high-quality data sets on threat-realistic targets against real backgrounds with state-of-the-art sensors; collecting global, seasonal statistics on stressing background clutter against the earth, earthlimb, and celestial backgrounds; and validating key sensor technologies such as focal planes, optics, and processors in operational environments over extended periods to support technology transfer.

MSX will also contribute significantly to the understanding of scientific issues of national interest, such as global change, (ozone chemistry, global warming, earth resources imagery), and basic science (astronomy, astrophysics and orbital debris, solar/terrestrial interactions, and celestial radiometric standards).

The MSX program will provide valuable insight into the operation of a tasked spacecraft, distributed data processing, and the efficient archive and retrieval of very large data sets. The infrastructure for experiment planning, operations, and data reduction and analysis is in place. Spacecraft integration is nearly complete and launch is planned for early 1996.

\section{REFERENCES}

[1] J. Mill, R. ONeill, S. Price, G. Romick, O. Uy, E. Gaposchkin, G. Light, W. Moore, T. Murdock, A.T. Stair $\mathrm{Jr}$,Midcourse Space Experiment: Introduction to the Spacecraft, Instruments, and Scientific Objectives, Journal of Spacecraft and Rockets, Volume 31, Number 5, September-October 1994.

[2] Richard Huebschman \& Tom Pardoe., "The Midcourse Space Experiment Spacecraft, "AIAA Paper 92-0977, Feb 1992.

[3] Midcourse Space Experiment "Program Management Plan", BMDO, Feb 1994.

[4] Midcourse Space Experiment Program, JHU/APL, Jan 1994.

[5] Harry Ames \& David Burt, Development of the SPIRIT III sensor, Proceeding of the SPIE conference on Cryogenic Optical Systems and Instruments VI, The International Society for Optical Engineering, Vol 2227, April 1994.

[6] D.C. Harrison \& J.C. Chow, Space-based visible sensor on the MSX satellite, Proceedings of the SPIE Conference on Surveillance Technologies, Vol 2217, April 1994. 


\section{Biography}

Bruce D. Guilmain is assigned to the Sensor and Phenomenology Technology Directorate, Ballistic Missile Defense Organization, Pentagon. As MSX Program manager, he leads a multi-service, multi-university team of more than 300 scientist and engineers.

Mr. Guilmain received a bachelor of science degree in electrical engineering from Lowell Technological Institute in Massachusetts in 1972. He earned a master of business administration degree in 1976 from the University of Montana and a masters degree in electrical engineering from the Air Force Institute of Technology at Wright Patterson Air force Base in 1978. Prior to his MSX assignment, Mr. Guilmain was Program Manager for Signal Processing Technologies, Strategic Defense Initiative Organization.

In 1989, as Chief, Special Activities Office, Headquarters Air Force Systems command, he was responsible for command management of the nation's highest priority classified space programs. In 1988 he was Chief of the Command, Control, and Communications Division at Wright Patterson AFB, responsible for all aspects of Foreign Technology Division's program for analyzing and reporting foreign $C^{3}$ systems. In 1981 he was assigned to Space Systems Division as Deputy Director field operations, responsible for onsite integration and testing of a newly designed command and control center for the Defense Meteorological Satellite Program. 